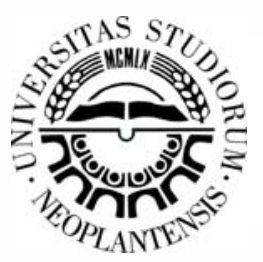

\title{
Rapid Prototyping of Art Sculptural Shapes According to the Sample
}

\author{
Saša Zivanović, ${ }^{a}{ }^{*}$ Slobodan Tabaković, ${ }^{b}$ Saša Randjelovićc \\ ${ }^{a}$ University of Belgrade, Faculty of Mechanical Engineering, Belgrade, Serbia \\ ${ }^{b}$ University of Novi Sad, Faculty of Technical Sciences, Novi Sad, Serbia \\ ${ }^{c}$ University of Niš, Faculty of Mechanical Engineering, Niš, Serbia
}

\begin{abstract}
The aim of this paper is to spread the use of Rapid Prototyping technology (RP) - Additive Manufacturing (AM), into design, restauration and making Art sculptural shapes according to the sample. Rapid prototyping is being used since its origin in designers' fields for a product design, and here it is presented in art design according to the sample, and it has been proved that it is a crucial tool in any design process. In this paper presents a procedure for the rapid prototyping of two models of monuments, the preparation of the model in the STL format and the making of scaled real models of the monuments.
\end{abstract}

Key words: rapid prototyping-additive manufacturing; art sculptural shape; programming; verification; simulation;

\section{INTRODUCTION}

Rapid prototyping (RP) is a technology for quickly fabricating physical models, also been referred to as additive manufacturing (AM), layer manufacturing, solid free-form fabrication, material addition manufacturing and 3D printing [1,2]. Rapid prototyping, in the first case, may be the additive technology that is using adding material in the layers. Another approach involves subtraction of materials, layer by layer [3].

Application of new technologies such as additive manufacturing, or subtraction of materials, layer by layer, which is based on models in STL format, is very interesting in the area of restoration art sculptural shapes [3] and archaeological artefacts [4]. It can also be interesting for sculptors, as a help for making sculptures according to the real model.

STL is a file format native to the stereolithography CAD software created by 3D Systems [5]. This file format is supported by many other software packages; it is widely used for rapid prototyping, 3D printing and computeraided manufacturing [6]. An example of *.stl file representation is shown on Fig.1. STL files use triangles facets or groups of triangles facets to approximate surfaces, Fig.2, where accuracy depends on the triangle sizes. Small triangle facets produce a high quality surface.

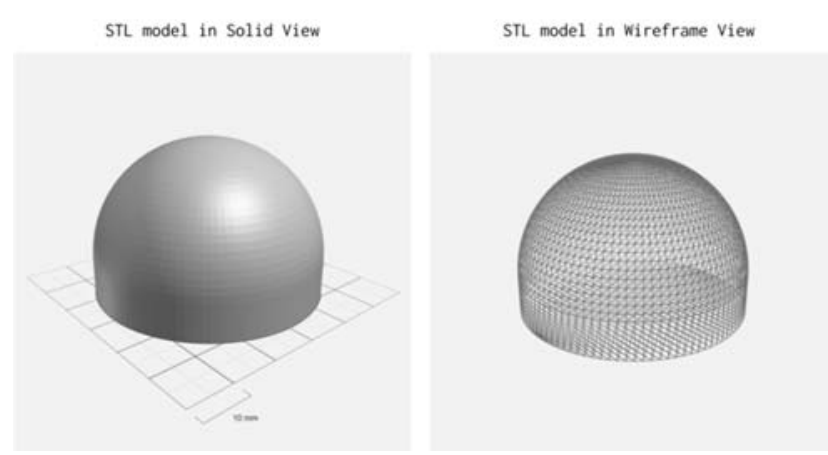

Fig. 1 An example of * ${ }^{*}$ stl representation

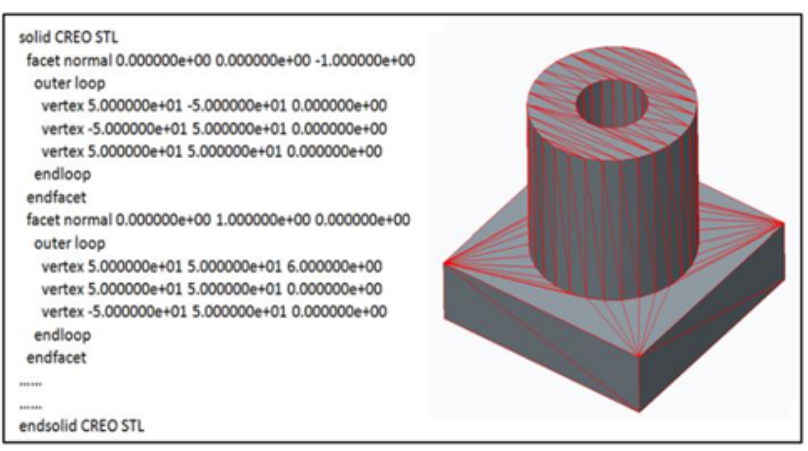

Fig. 2 Format of STL file for one example 
With the rapid development of computers and software for the CAD product design and $3 \mathrm{D}$ modelling of the sculptural shapes is rapidly progressed, including technology to produce realistic physical models.

3D models of the sculptural shapes, monuments and archaeological artefacts can be obtained in several ways: (i) using specialized software for modelling; (ii) obtaining a $3 \mathrm{D}$ scanning point cloud data for the model, using the reverse engineering method for getting a CAD model; (iii) using photogrammetric method, which is based on 2D photos on the basis of which the obtained 3D CAD model. The third mentioned way is the main topic of this paper.

The outputs from the research of this topic should prove to be valuable to anyone working in the field of digital restoration, particularly when digital capture of shape based on photos and the creation of physical replicas are required. For illustration of obtaining the model for art sculptural shapes, real models were selected.

As example for illustration how to prepare the model in STL format based on 2D photos using appropriate software, in this paper use of the monument of serbian military commander Petar Bojovic, Fig.3.

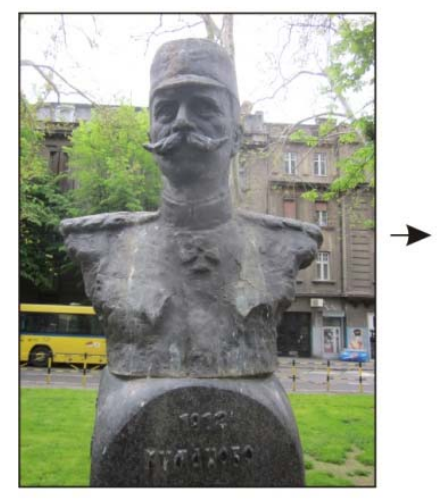

Art sculptural shapes

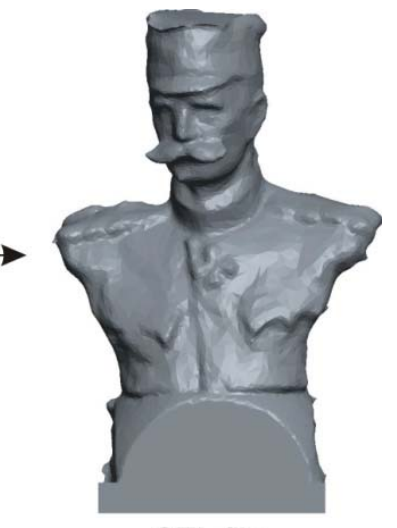

STL file
Fig. 3 CAD model of the monument and obtained STL model

Now there are already many manufacturers of equipment for rapid prototyping, so there are a lot of different technologies. Classification of RP technologies can be made upon different criteria. More detailed information on every specific RP method can be found in the references [1-3].

Rapid prototyping is a typical additive technology, where models are formed layer by layer. Typical process stages for additive manufacturing is shown in Fig. 4 and includes the following stages: (i) CAD modelling of part, (ii) STL file is exported from CAD model and STL file is checked for defects, (iii) STL files are used as an input to slicing software, (iv) Machine setup, (v) simulation of 3D printing, (vi) building prototype, (vii) additional interventions on prototype (3D printed model) such as curing, surface polishing, finishing, etc.

In the next sections, this procedure is applied in the case of the realization of the physical model of monument from Fig.3. In section 3 are shown programming, verification and simulation for additive manufacturing technology on the example from Fig.3.

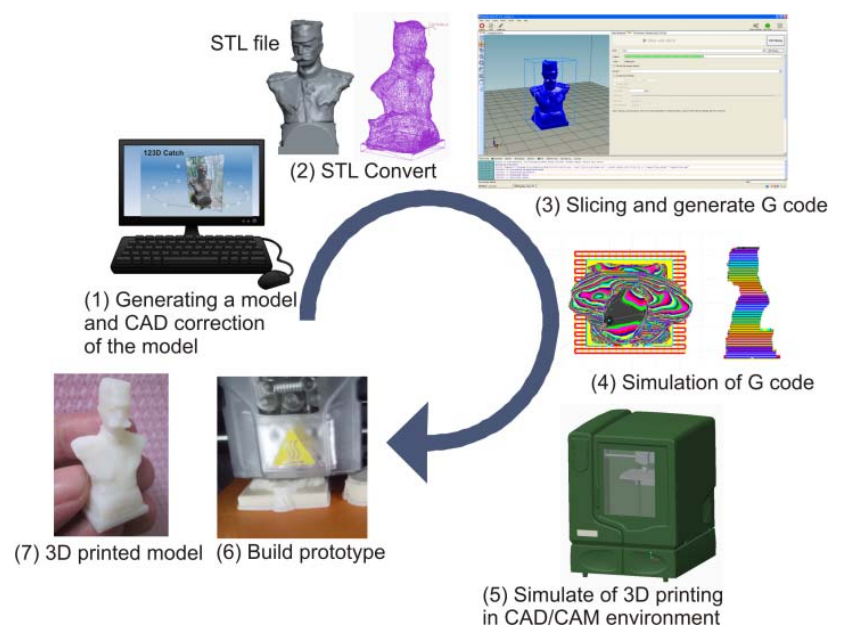

Fig. 4 Stages for additive manufacturing process from $C A D$ model to 3D part

\section{PREPARE OF ART SCUIPTURAL SHAPES ACCORDING TOTHE SAMPLE}

For the preparation of sculptural art shapes for additive manufacturing it is necessary to obtain model in STL format. In this case, the STL model obtained by photogrammetric method based on 2D photo [3] using the software Autodesk 123D Catch [7] Fig.5.

\subsection{Autodesk 123D Catch}

123D Catch is a handy, free Windows program, belonging to the category Design \& photography software with subcategory 3D Design and has been published by Autodesk [7]. This software can working on the PC, web, tablet or mobile phone platform for digital sculpting.

123D Catch allows creating 3D models from series of photographs taken at various angles using photogrammetric method. Examples of recorded photographs for the model from Fig.3, are shown in the Fig 5a.

The nicest feature of $123 \mathrm{D}$ Catch (on mobile phone or tablet platform) is that it guides you through the phototaking process by using the gyroscope in your phone to determine which angles you've done. The indicator shows two 360-degree indicators: one for taking a series of 18 photos straight at your object and additional 6 photos from a downwards-facing angle. The indicator can be switched off, allowing you to take up to 70 photos from any angle you like.

The photos obtained in this way are loaded into the 123D Catch software, where photos are analyzed and possibly excluded photos of poor quality. The shooting plan can be seen in the software, Fig 3b. Software generates a 3D model in STL format, on a computer or on a Cloud platform. After we get the initial STL model, thus obtained file is not perfect and needs to be analyzed, cleaned out and corrected if some of the surface areas are 
not complete. When model is finished in the CAD system, need prepare final STL format for next step.
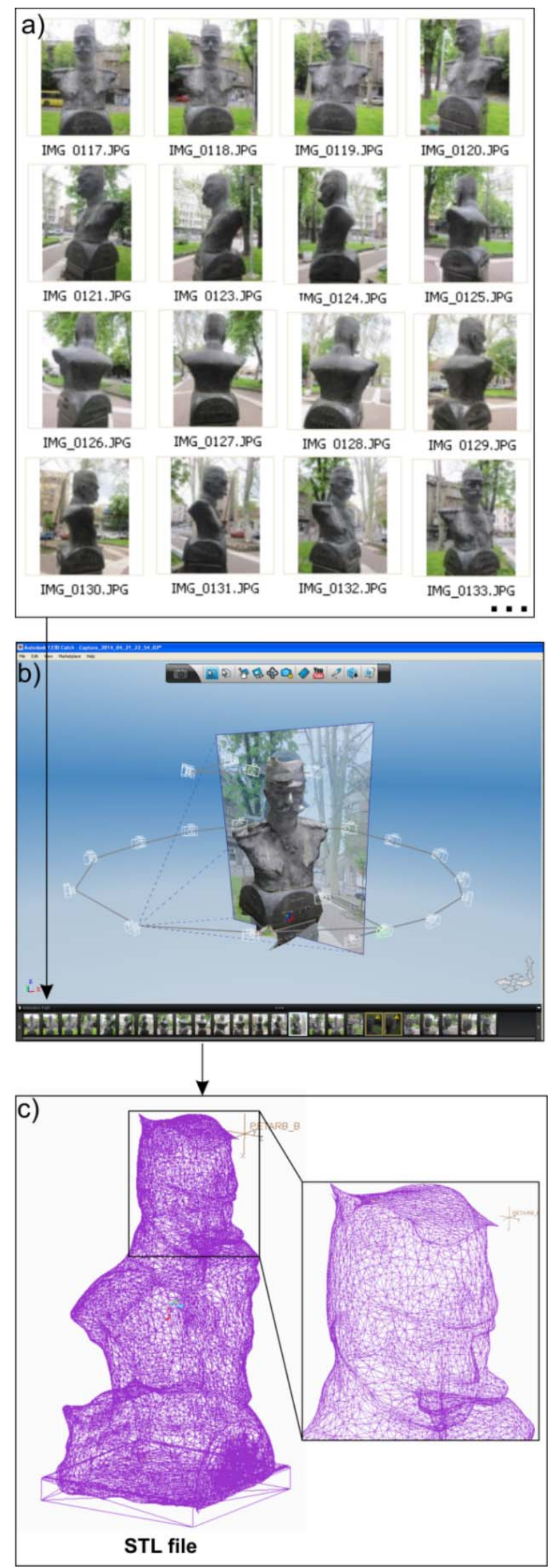

Fig. 5 Prepare art sculpture shape using 123D Catch software
The successor of the Autodesk 123D Cath software is Autodesk ReCap Photo, which is an extension of Autodesk ReCap Pro made for converting aerial and object photographs to 3D models [8].

\subsection{Autodesk 123D Make}

Autodesk 123D Make [9], is licensed as Freeware for Windows operating system without restrictions. Software allows creation of low-tech LOM (Laminated Object Manufacturing) - style solid models. Autodesk 123D Make loaded STL model based on which work slicing and preparation of layers for additive construction [3,9]. Realization of art sculpture shape, from Fig.3 using 123D Make software, shown in Fig.6
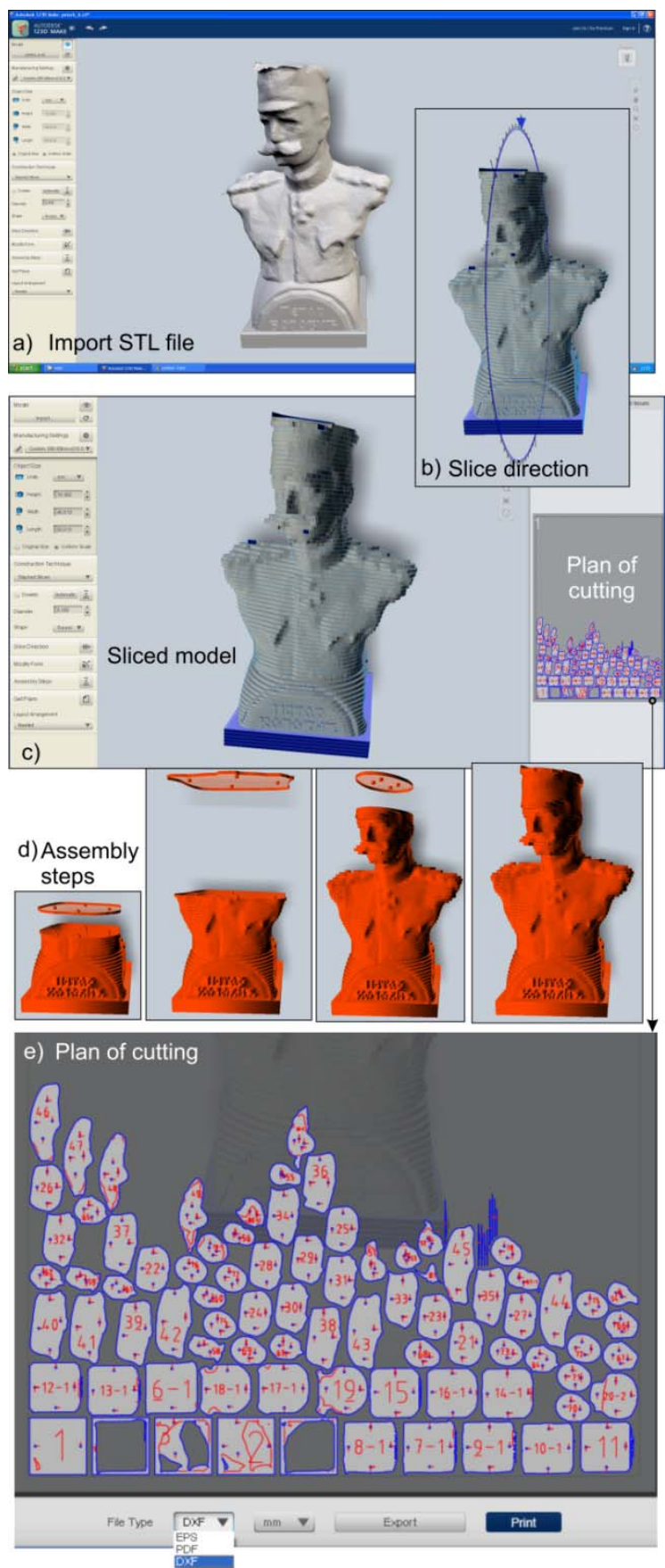

Fig. 6 Realization of art sculpture shape using 123D Make software 
To use Autodesk 123D Make, you should load the model (in STL format) into the program and choose the type of construction you would like to create the object with. You can import designs from other programs. In this case used type of construction of adding parallel layers according to the base of model (Stacked slices).

For a loaded model, it is possible to choose the Slice direction, which is most appropriate for building of physical model. Setting the basic parameters for building the model is done in the Manufacturing settings, where it is most important to choose the Thickness of layer. The plan for cutting all the layers is done by choosing the Get Plans option.

It is possible to choose two options for schedule all layers: Simple and Nested layers. Nested is a better option because it performs the optimal schedule of the layers on the cutting board, as shown in Fig. 6e.

In this software can applying various slicing techniques to your model and create 2D plans in EPS, DXF or PDF formats, Fig. 6e, that you can cut using Laser cutter or CNC machine.

Software has also the possibility of simulation the adding layer by layer, using option Assembly Steps, Fig 4d. The illustration of this kind of additive manufacturing was shown Fig 6.

This program is now known as Slicer for Autodesk Fusion 360 [10]. Slicer for Fusion 360, will allow you to make incredible and complex designs, based on the 123D Make technology. Slicer for Autodesk Fusion 360 is software tools which can slices and converts 3D models into 2D patterns that you can cut out of any flat material.

\section{PROGRAMMING, VERIFCATION AND SIMULATION OF ADDITIVEMANUFACTURING}

Procedure for building the 3D physical model of the art sculptural shape using Fused deposition modelling (FDM) method, is shown on Fig.7.

This procedure including: (i) input of STL file in appropriate software for additive manufacturing, (ii) slicing of STL model and obtain G Code for each layer, (iii) verification of obtained $G$ code, using simulation option of software, or specialized software for simulation , (iv) sending verified $\mathrm{G}$ code to 3D printing.

The obtained G code based on STL file, it is possible to verify using: specialized software like Gcode Print Simulator [11], (ii) commonly CNC editors for simulating the tool path, CIMCO [12], (iii) WEB-CNC simulator which include 3D printing [13].

Based on the input of the STL file, the G code was postprocessed, and then the simulation of additive manufacturing was performed, in order to finally make a physical model on the 3D printer Stratasys uPrint SE. In order to send the STL model to the 3D print, the software Catalyst EX is used as an interface for communicating with the available printer and which is a recommendation or basic communication software for printers produced by firm Stratasys [3].

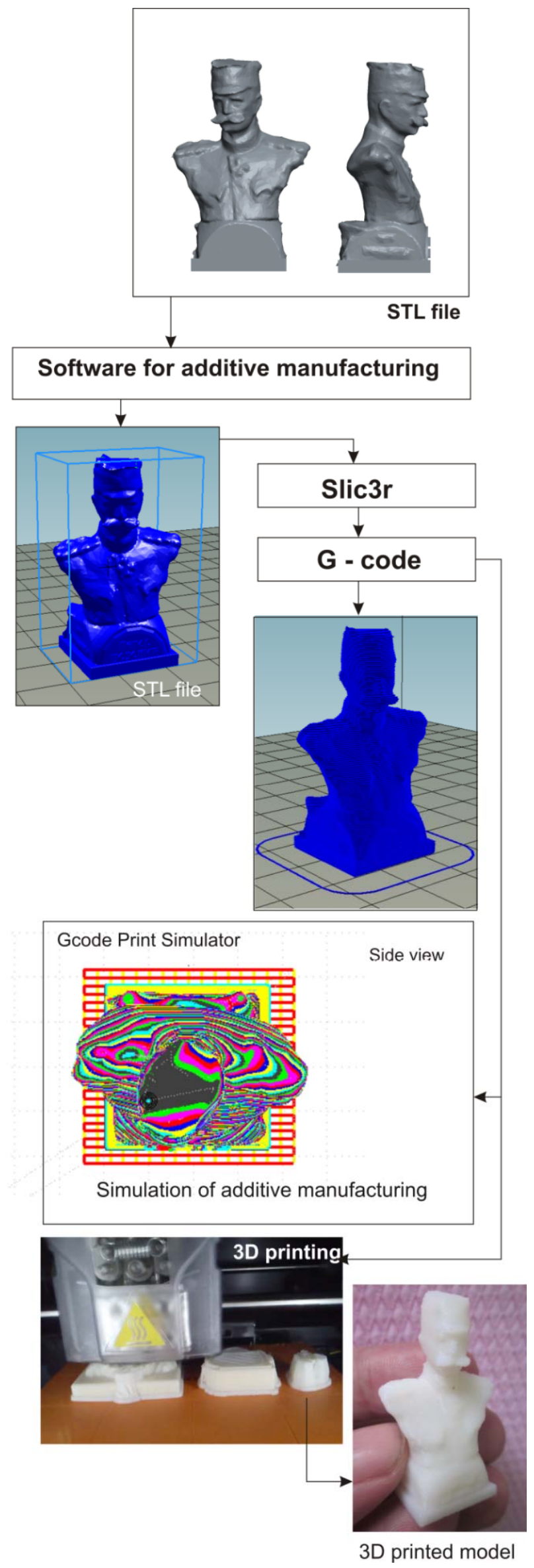

Fig. 7 Building the 3D model of the sculpture using FDM method 


\subsection{Machine simulation of 3D printing in CAD/CAM environment}

Today and commercial CAD/CAM systems are beginning to integrate Additive technology into theirs CAM modules. For example, in a CAD/CAM system PTC Creo [14] from version 3.0 M040 have Additive Manufacturing functionality.

A standard characteristic of most CAD/CAM systems is the simulation of the virtual machine tool along a given tool path. For now, there is no possibility of direct simulation of the virtual machine for additive manufacturing, although this is possible realize an indirect way in PTC Creo. This indirect method implies that the $3 \mathrm{D}$ printer is modelled in the same way as the milling machine with the same kinematics [3].

Machine tools for additive manufacturing, or as they are also called 3D printers, can be modelled as a CAD model for configuring virtual 3D printer, which includes appropriate kinematic connections. The most common machine tool for additive manufacturing is machine with serial kinematics and three translatory axes. Each of the translatory axes is defined as kinematic connection type Slider. During the simulation, the machine is treated as if the milling machine, that moves along a path of adding material. An example of this kind of simulation is shown on Fig.8. and detailed explanation can be seen in the previous paper [3].

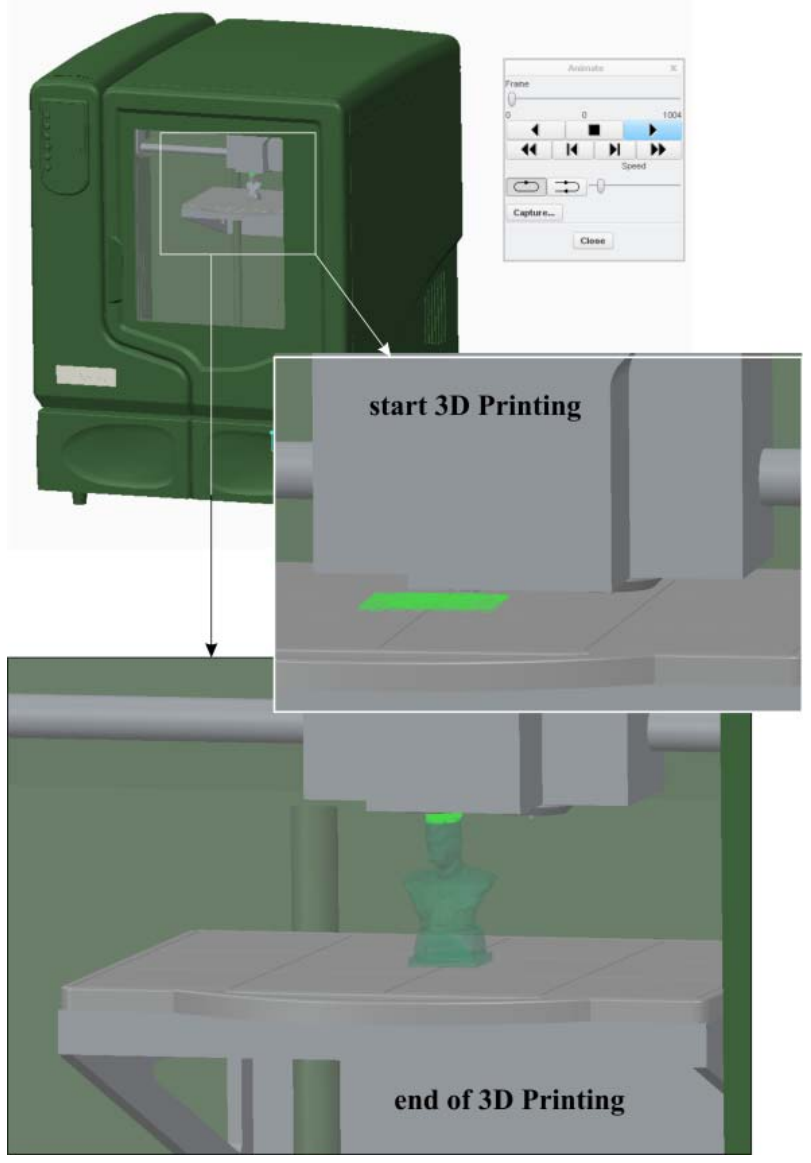

Fig. 8 Machine simulation in PTC Creo environment for toolpath of 3D printing
To obtain the path of adding material, it is necessary to convert the path of the printing head into $G$ code. Thus obtained path in $\mathrm{G}$ code can be converted into the DXF file using appropriate software (CIMCO [12]). The obtained DXF file is loaded into the CAM module where the tool path for the simulation is generated. Tool path is generating using the Custom Trajectory strategy. After that, is possible simulate tool-path for additive manufacturing in CAD/CAM environment.

During the simulation, we have the ability to load CAD model of the machine (3D printer), which is made for simulation purposes and better visualization, as shown in Fig. 9. This kind of simulation is especially important for the $3 \mathrm{D}$ printers with parallel kinematics.

Examples of the additive manufactured parts obtained on an illustrated manner using the FDM are shown in Fig. 9. These models are: b) the sculpture of the author, c) the monument to the fighters of the First World War in Paracin and d) the sculpture of the serbian military commander Petar Bojovic.

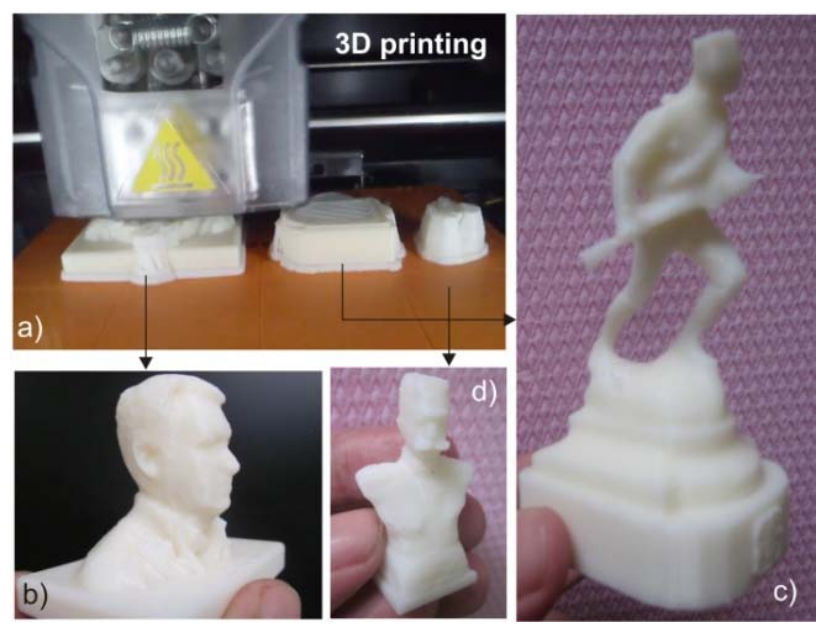

Fig. 9 Examples of the additive manufactured parts

\section{CONCLUSIONS}

In this paper is explained a photogrammetric method of obtaining STL model, based on 2D photos, using software Autodesk 123D Catch.

The paper presents the procedures for obtaining physical model of art sculptural shapes on two ways: (i) using software 123D Make, and (ii) using classical software for 3D printing and FDM method.

The paper analyzes the possibility of tool-path simulation by adding a material, as well as the simulation of the machine for additive manufacturing ("3D printers").

This research should be valuable to anyone working in the field of digital restoration and fine art sculpture particularly when digital capture of shape based on photos and the creation of physical replicas are required.

In the future work is planned preparation of new more complex models based on 2D photos, as well as path simulation by adding a material for additive manufacturing, using "3D printers" with parallel and/or hybrid kinematics. 


\section{ACKNOWHEDENENTS}

The author would like to thank the Ministry of Education, Science and Technological Development of Serbia for providing financial support that made this work possible.

\section{RefERENCES}

[1] Pham, D., Dimov, S. (2001). Rapid Manufacturing: The Technologies and Applications of Rapid Prototyping and Rapid Tooling, Springer Verlag, London.

[2] Pham, D., Dimov, S. (2003). Rapid prototyping and rapid tooling - the key enablers for rapid manufacturing. Proceedings of the Institution of Mechanical Engineers, Part C, 217, pp 1-23.

[3] Zivanovic, S. (2017). Rapid prototyping and manufacturing for model of human head. Proceedings of the 8th International Scientific Conference IRMES 2017, Trebinje, Bosnia and Herzegovina, pp. 271276.

[4] Zhang, F., Campbell, R.I., Graham, I.J. (2016). Application of additive manufacturing to the digital restoration of archaeological artefacts, Int. J. Rapid Manufacturing, 6(1), 75-94.
[5] 3D Systems, from www.3dsystems.com, accessed on 2019-1-11.

[6] Chua, C. K., Leong, K. F., Lim, C. S. (2003). Rapid Prototyping: Principles and Applications (2nd ed.), World Scientific Publishing Co.

[7] Autodesk 123D Catch, from https://autodesk-123datch.en.softonic.com/, accessed on 2018-07-06.

[8] RECAP, from https://www.autodesk.com/products/recap/overview, accessed on 2018-12-11.

[9] Autodesk 123D Make, from http://autodesk-123dmake.en.lo4d.com/, accessed on 2018-07-06.

[10] Slicer for Autodesk Fusion 360, from https://www.autodesk.com/products/fusion360/blog/quick-tip-slicer-fusion-360/, accessed on 2018-12-11.

[11] Gcode Print Simulator, from https://www.thingiverse.com/thing:44286, accessed on 2018-07-14.

[12] CICMO, from http://www.cimco.com/, accessed on 2018-07-13.

[13] CNC WEB Simulator, from http://filipecaixeta.com.br/cncwebsim/, accessed on 2018-07-14.

[14] PTC, Creo Parametric, from http://www.ptc.com/cad/creo/parametric, accessed on 2018-07-13. 